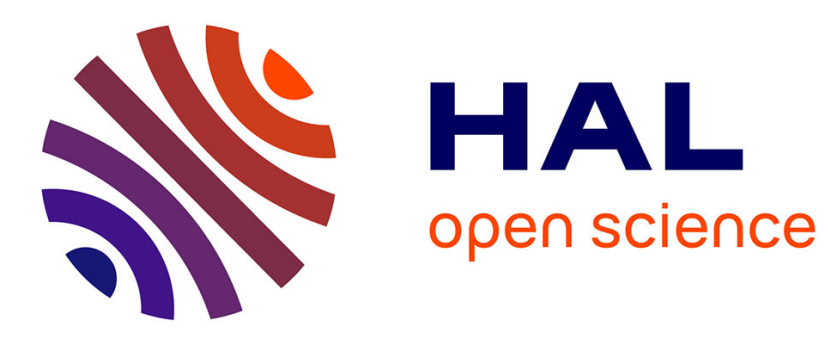

\title{
Program Calculation in Coq
}

Julien Tesson, Hideki Hashimoto, Zhenjiang Hu, Frédéric Loulergue, Masato

Takeichi

\section{To cite this version:}

Julien Tesson, Hideki Hashimoto, Zhenjiang Hu, Frédéric Loulergue, Masato Takeichi. Program Calculation in Coq. [Research Report] RR-2009-07, 2009, pp.18. inria-00448751

\section{HAL Id: inria-00448751 \\ https://hal.inria.fr/inria-00448751}

Submitted on 20 Jan 2010

HAL is a multi-disciplinary open access archive for the deposit and dissemination of scientific research documents, whether they are published or not. The documents may come from teaching and research institutions in France or abroad, or from public or private research centers.
L'archive ouverte pluridisciplinaire HAL, est destinée au dépôt et à la diffusion de documents scientifiques de niveau recherche, publiés ou non, émanant des établissements d'enseignement et de recherche français ou étrangers, des laboratoires publics ou privés. 


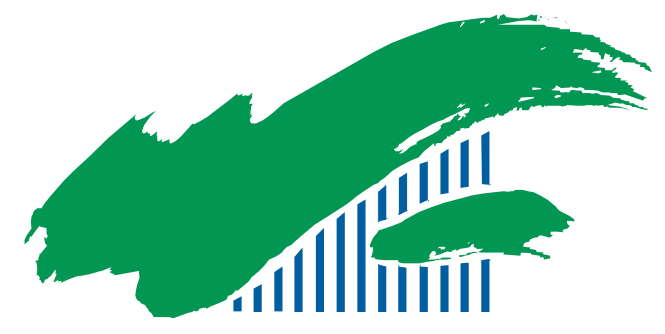

UNIVERSITE D'ORLEANS

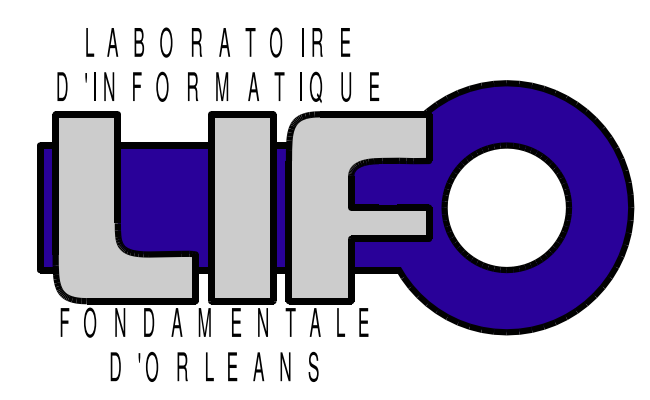

4 rue Léonard de Vinci

BP 6759

F-45067 Orléans Cedex 2

FRANCE

http://www.univ-orleans.fr/lifo

\section{Rapport de Recherche}

\section{Program Calculation in Coq}

Julien Tesson, Hideki Hashimoto, Zhenjiang Hu, Frédéric Loulergue, and Masato Takeichi

Rapport n ${ }^{o}$ RR-2009-07 


\title{
Program Calculation in Coq
}

\author{
Julien Tesson ${ }^{1}$, Hideki Hashimoto ${ }^{2}$, \\ Zhenjiang $\mathrm{Hu}^{3}$, Frédéric Loulergue ${ }^{1}$, and \\ Masato Takeichi ${ }^{2}$ \\ 1 Université d'Orléans, LIFO, France \\ \{julien.tesson, frederic.loulergue\}@univ-orleans.fr \\ 2 The University of Tokyo, Japan \\ \{hhashimoto, takeichi\}@ipl.t.u-tokyo.ac.jp \\ 3 National Institute of Informatics, Tokyo, Japan \\ hu@nii.ac.jp
}

\begin{abstract}
Program calculation, being a programming technique that derives programs from specification by means of formula manipulation, is a challenging activity. It requires human insights and creativity, and needs systems to help human to focus on clever parts of the derivation by automating tedious ones and verifying correctness of transformations. Different from many existing systems, we show in this paper that Coq, a popular theorem prover, provides a cheap way to implement a powerful system to support program calculation, which has not been recognized so far. We design and implement a set of tactics for the Coq proof assistant to help the user to derive programs by program calculation and to write proofs in calculational form. The use of these tactics is demonstrated through program calculations in Coq based on the theory of lists.
\end{abstract}

\section{Introduction}

Programming is the art of designing efficient programs that meet their specifications. There are two approaches. The first approach consists in constructing a program and then proving that the program meets its specification. However, the verification of a (big) program is rather difficult and often neglected by many programmers in practice. The second approach is to construct a program and its correctness proof hand in hand, therefore making a posterior program verification unnecessary.

Program calculation [1-3], following the second approach, is a new style of programming technique that derives programs from specification by means of formula manipulation: calculations that lead to the program are carried out in small steps so that each individual step is easily verified. More concretely, in program calculation, specification could be a program that straightforwardly solve the problem, and it is rewritten into a more and more efficient one without changing the meaning by application of calculation rules (theorems). If the program before transformation is correct, then the one after transformation is guaranteed to be correct because the meaning of the program is preserved by the transformation.

Bird-Meertens Formalism (BMF) [1,4], proposed in late 1980s, is a very useful program calculus for representing (functional) programs, manipulating programs through equational reasoning, and constructing calculation rules (theorems). Not only many general theories such as the theory of list [4] and the theory of trees [5] have been proposed, but also a lot of useful specific theories have been developed for dynamic programming [6], parallelization [7], etc.

Program calculation with BMF, however, is not mechanical: it is a challenging activity that requires creativity. As a simple example, consider that we want to develop a program 
that compute the maximum value from a list of numbers and suppose that we have had a program to sort a list. Then a straightforward solution to the problem is to sort a list and then get the first element:

$$
\text { maximum }=h d \circ \text { sort } .
$$

However, it is not efficient because it takes at least the time of sort. Indeed, we can calculate a linear program from this solution by induction on the input list.

If the input is a singleton list $[a]$, we have

$$
\begin{aligned}
& \text { maximum }[a] \\
= & \{\text { def. of maximum }\} \\
& (h d \circ \text { sort })[a] \\
= & \{\text { def. of function composition }\} \\
& h d(\text { sort }[a]) \\
= & \{\text { def. of sort }\} \\
= & h d[a] \\
= & a \text { def. of hd }\}
\end{aligned}
$$

Otherwise the input is a longer list of the form $a:: x$ whose head element is $a$ and tail part is $x$, and we have

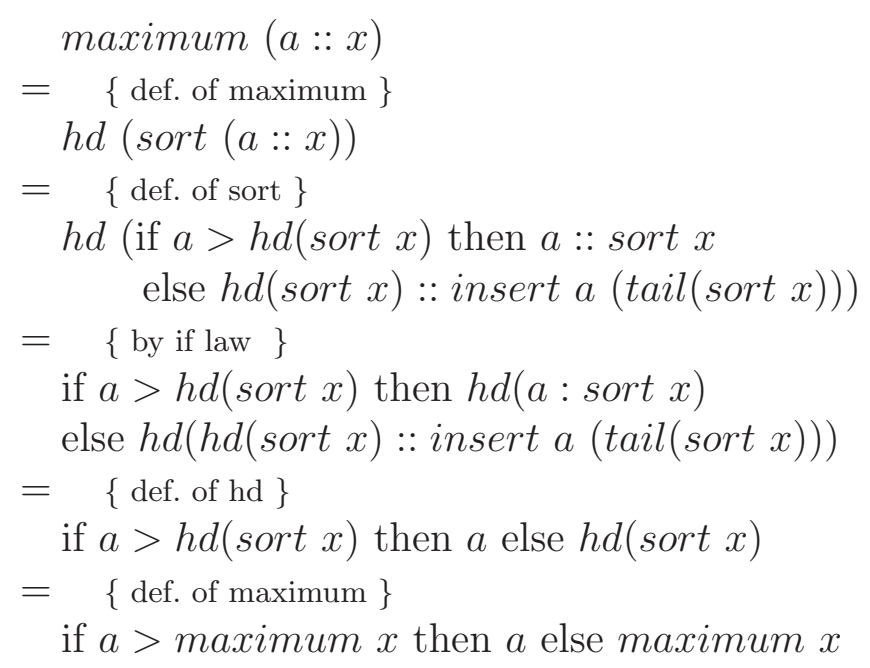

Consequently we derive the following linear program:

$$
\begin{aligned}
& \operatorname{maximum}[a]=a \\
& \operatorname{maximum}(a:: x)=\text { if } a>\text { maximum } x \text { then } a \text { else maximum } x
\end{aligned}
$$

In this derivation, we transform the program by equational reasoning via unfolding definition of functions and applying some existing calculation laws (rules). Sometimes, we even need to develop new calculations to capture important transformation steps. This calls for an environment, and much effort has been devoted to development of systems to support correct and productive program calculation. Examples are KIDS [8], MAG [9], Yicho [10], and so on. In general, this kind of environments should (1) support 
interactive development of programs by equational reasoning so that users can focus on his/her creative steps, (2) guarantee correctness of the derived program by automatically verifying each calculation step, (3) support development of new calculation rules so that mechanical derivation steps can be easily packed, and (4) make development process easy to maintain (i.e., development process should be well documented.). In fact, developing such a system from scratch is hard and time-consuming, and there are few systems that are really widely used.

The purpose of this paper is to show that Coq [11], a popular theorem prover, provides a cheap way to implement a powerful system for program calculation, which has not been recognized so far. Coq is an interactive proof assistant for the development of mathematical theories and formally certified software. It is based on a theory called the calculus of inductive constructions, a variant of type theory. Although little attention has been paid on using Coq for program calculation, Coq itself is indeed a very powerful tool for program development. First, we can use dependent type to describe specifications in different levels. For instance, we can write the specification for sort by

$$
\text { sort : } \forall x: \text { list nat, } \exists y: \text { list nat, }(\operatorname{sorted}(y) \wedge \operatorname{permutation}(x, y))
$$

saying that for any $x$, a list of natural numbers, there exists a sorted list $y$ that is a permutation of $x$, and we may write the following specification for maximum.

$$
\text { maximum }: \exists \oplus: \text { nat } \rightarrow \text { nat } \rightarrow \text { nat }, \text { hd } \circ \text { sort }=\text { foldr1 }(\oplus)
$$

saying that the straightforward solution hd o sort can be transformed into a foldr1 program. Second, one can use Coq to describe rules for equational reasoning with dependent types again. Here are two simple calculation rules, associativity of the append operation, and distributivity of the map functions.

$$
\begin{aligned}
& \text { Lemma appAssoc: } \forall(A: \text { Type })(l m n: \text { list } A), \\
& \qquad(l+m)+n=l+(m+n) \\
& \text { Lemma mapDist }: \forall(A B C: \text { Type })(f: B \rightarrow C)(g: A \rightarrow B), \\
& \text { map } f \circ \text { map } g=\operatorname{map}(f \circ g)
\end{aligned}
$$

Third, one can use Coq to prove theorems and extract programs from the proofs. For example, one can prove the specification for maximum in Coq as in Section 2. The proof script, however, is usually difficult to read compared with the calculation previously introduced. This is one of the main problem of using Coq for program calculation.

In this paper, we shall report our first attempt of designing and implementing a Coq tactic library (of only about 200 lines of Tactic codes), with which one can perform correct program calculations in Coq, utilize all the theories in Coq for his calculation, and develop new calculation rules, laws and theorems. Section 3 shows an example of the calculation for maximum in Coq. A more interesting use of these tactics are demonstrated through implementing the Bird's calculational theory of lists in Coq. All the codes of the library and applications are available at the following web page:

https://traclifo.univ-orleans.fr/SDPP 
The organization of the paper is as follows. After a very short introduction to Coq in Section 2, we discuss the design and implementation of a set of tactics for supporting program calculation and writing proofs in calculational form in Section 3. Then, we demonstrate an interesting application of program calculations on lists in Section 4. Finally, we discuss the related work in Section 5 and conclude the paper in Section 6.

\section{A Very Short Introduction to Coq}

The Coq proof assistant [12] is based on the calculus of inductive constructions. This calculus is a higher-order typed $\lambda$-calculus. Theorems are types and their proofs are terms of the calculus. The Coq systems helps the user to build the proof terms and offers a language of tactics to do so.

We illustrate quickly all these notions on a short example :

\section{Set Implicit Arguments.}

Inductive list (A:Set) : Set :=

| nil : list $A$

cons : $A \rightarrow$ list $A \rightarrow$ list $A$.

Implicit Arguments nil $[\mathrm{A}]$.

The Set Implicit Arguments command indicates that we let the Coq system infer as many arguments as possible of the terms we define. If in some context the system cannot infer the arguments, the user has to specify them. In the remaining of this short introduction, the Coq system always infers them.

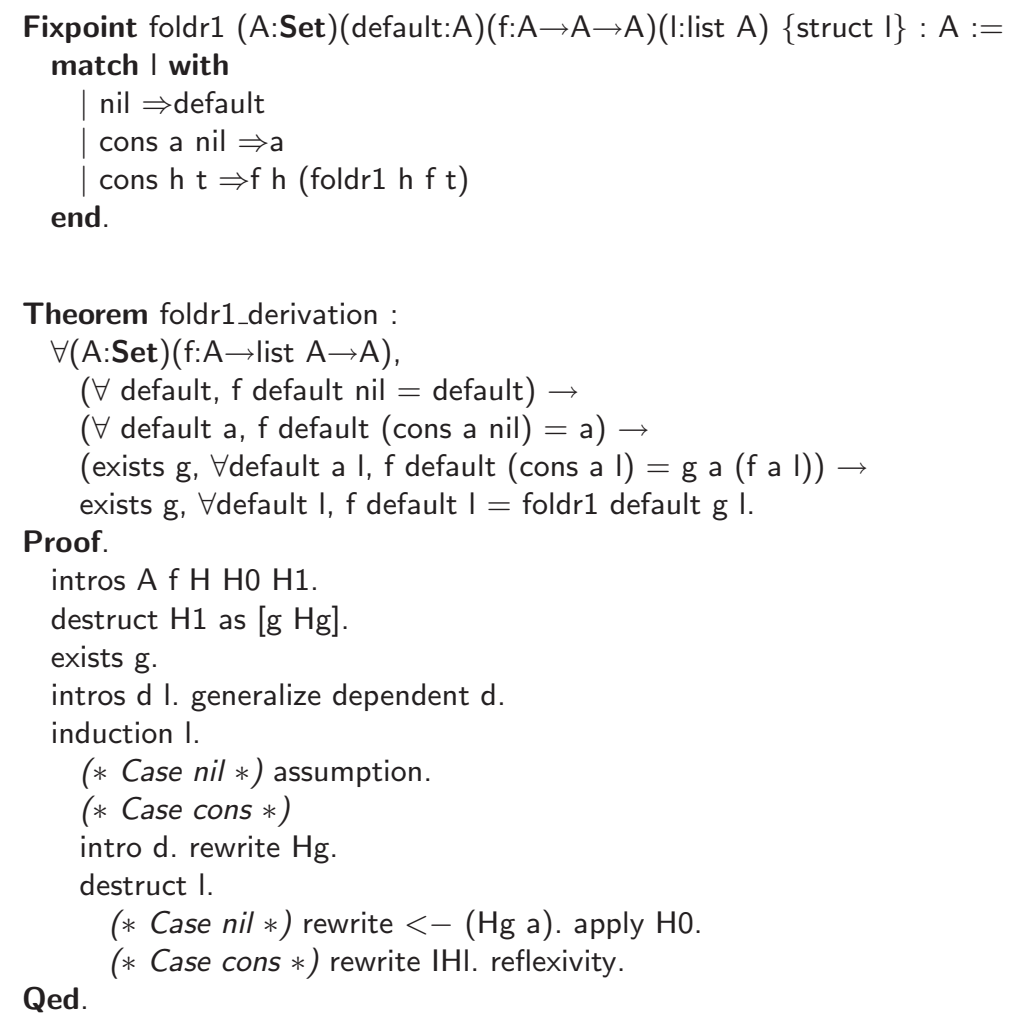




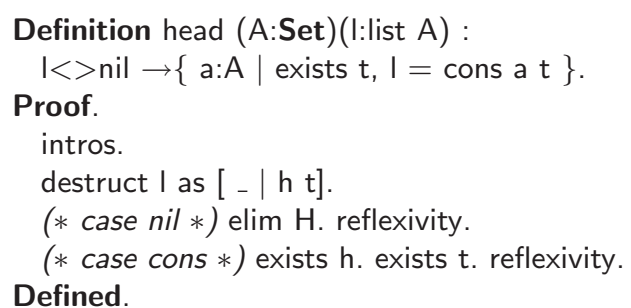

\section{Extraction head.}

In this example, we first define a new inductive type, the type of lists. This type is a polymorphic type since it takes as an argument a type A, the type of the elements of the list. A has type set which means it belongs to the computational realm of the Coq language similar to ML data structures. The definition of this new type introduces two new functions, the constructors of this type: nil and cons. Then we define a recursive function: foldr1. In this definition we specify the decreasing argument (here the fourth argument) as all functions must be terminating in Coq and we give its type (after ":") as well as a term (after ":=") of this type.

Note that usually foldr1 is undefined for the empty list. However in Coq, all functions are total. Thus there are several ways to model a partially defined function in Coq. One possible solution is to have an additional argument which is a default value in case the function is applied to the empty list. Compared to the "paper" or functional programming language versions, we have to deal with this extra parameter. Another approach is to define a function that returns an optional value, ie a value of the type:

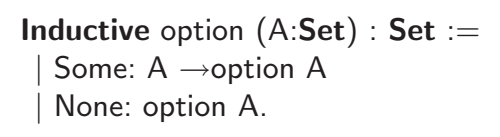

A third possibility is to take as extra argument a proof that the list is non empty. It is even possible to create a new type of non-empty lists, an element of this type being a list together with a proof that this list is not nil. In the example we chose the first solution.

We then define a theorem named foldr1_derivation stating that:

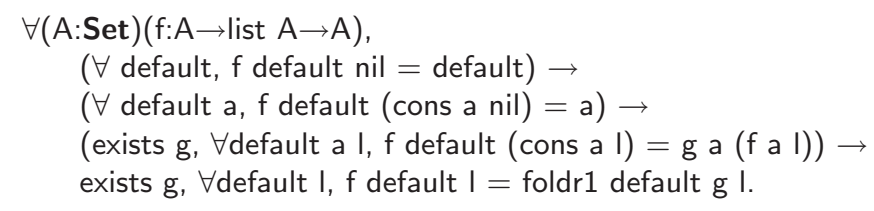

If we check (using the Check command of Coq) the type of this expression, we would obtain Prop meaning that this expression belongs to the logical realm. To define foldr1_derivation we also should provide a term of this type, that is a proof of this theorem. We could write directly such a term, but it is usually complicated and Coq provides a language of tactics to help the user to build a proof term. If we give to Coq top-level the line beginning with Theorem we would enter the interactive proof mode. We should prove the goal:

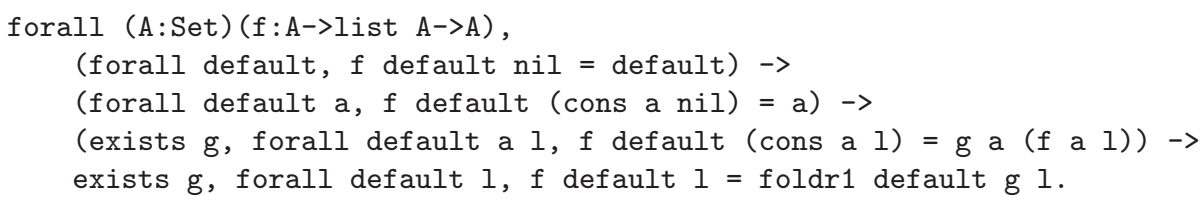


After the command Proof., one can use tactics to build step by step a term of the given type. The first tactic intros is used to move premises or universally quantified variables from the goal to the context. The new goal (with its context) is thus:

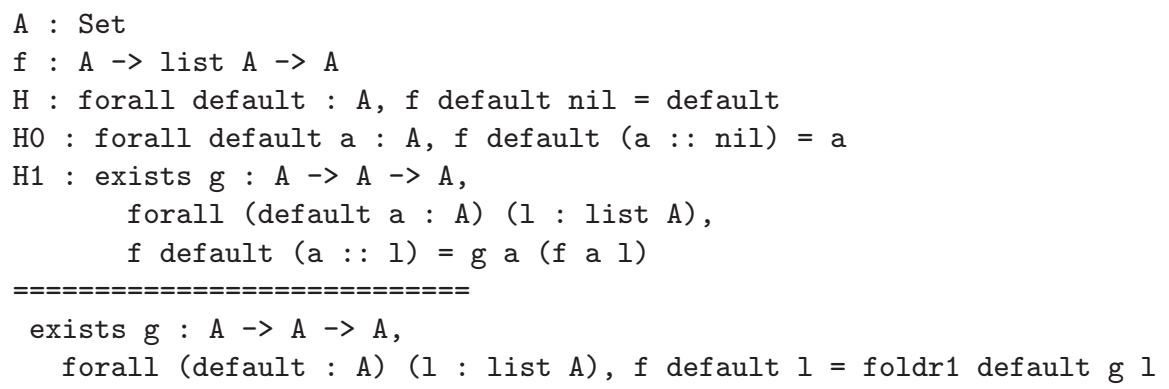

To prove an existential statement, one has to provide a witness. In our case, the hypothesis $\mathrm{H} 1$ states the existence of a function that is what we need. We can use the tactic destruct to decompose the hypothesis $\mathrm{H} 1$ into a function $\mathrm{g}$ and an hypothesis that states its property. Then, with the tactic exists, we can provide this function $\mathrm{g}$ as a witness, leading to the goal:

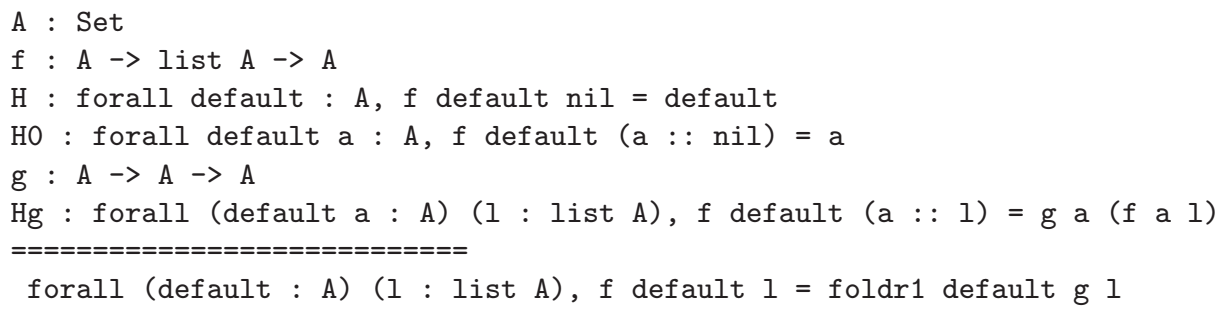

Now we can proceed by reasoning by induction on the list I. However, we would like the induction hypothesis to be universally quantified over default. Thus we should introduce d and I, then generalize the variable d before using the tactic induction, to obtain two goals, one for each constructor of list:

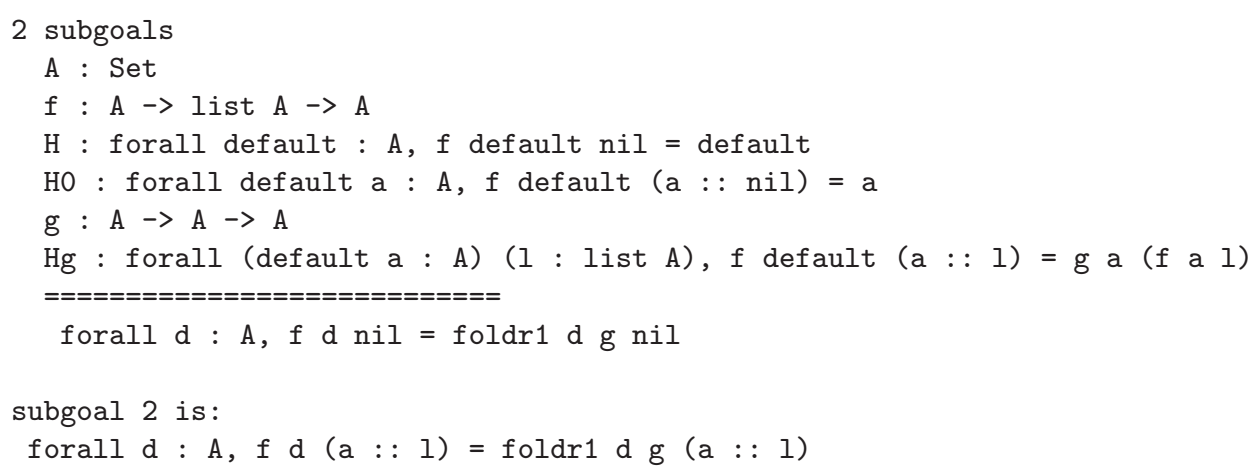

The first subgoal is immediately solved using the assumption $\mathrm{H}$. To prove the second subgoal, we decompose again the list, but there is no need for induction: we reason by case analysis on the list using the tactic destruct. To solve the first new subgoal we rewrite $g a(f a$ nil) in $f a$ (cons a $l$ ) using the equality (from right to left) provided by the hypothesis $\mathrm{Hg}$ (applied to a). We can conclude by applying hypothesis H0. The second new subgoal is also proved with the tactic rewrite but with the equality (from left to right) provided by the induction hypothesis $\mathbf{I H I}$ (we can see that it was important to have an 
hypothesis universally quantified). The obtained goal is an equality with both sides equal after reduction: it can be solved with reflexivity.

Mixing logical and computational parts is possible in Coq. For example a function of type $\mathrm{A} \rightarrow \mathrm{B}$ with a precondition $\mathrm{P}$ and a postcondition $\mathrm{Q}$ corresponds to a constructive proof of type: $\forall x: A,(P \times) \rightarrow$ exists $y: B \rightarrow(Q \times y)$. This could be expressed in Coq by using the inductive type sig:

Inductive sig (A:Set) $(\mathrm{Q}: A \rightarrow$ Prop $):$ Set $:=\mid$ exist: $\forall(x: A),(Q x) \rightarrow(\operatorname{sig} A$ Q $)$.

It could also be written, using syntactic sugar, as $\{\mathrm{x}: \mathrm{A} \mid(\mathrm{Q} \times)\}$.

This feature is used in the definition of the function head. The specification of this function is: $\forall($ A:Set $)(\mathrm{I}:$ list $\mathrm{A}), \mathrm{I}<>$ nil $\rightarrow\{$ a:A $\mid$ exists $\mathrm{t}, \mathrm{I}=$ cons a $\mathrm{t}\}$ and we build it using tactics. We reason by case on I (tactic destruct). The first case is easily solved because we have the hypothesis nil<>nil, the second one only needs to state that the head and tail of I are the required values.

The command Extraction head would extract the computational part of the definition of head. We could obtain a certified implementation of the head function:

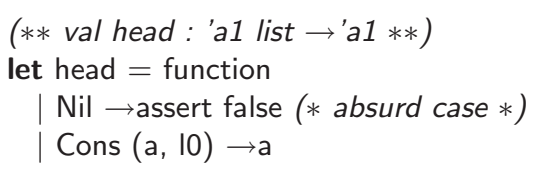

Let us see how we can deal with the maximum example presented in introduction:

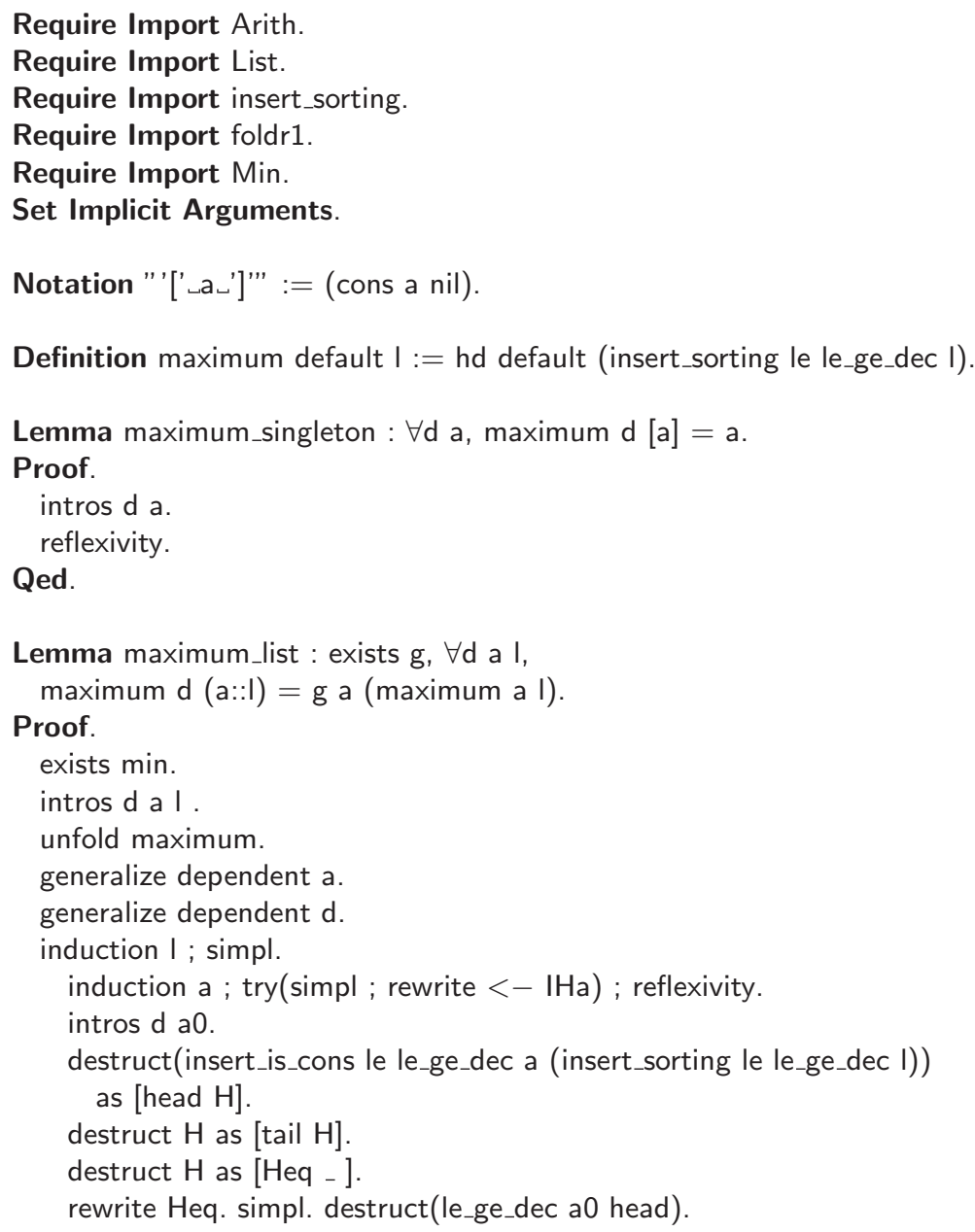




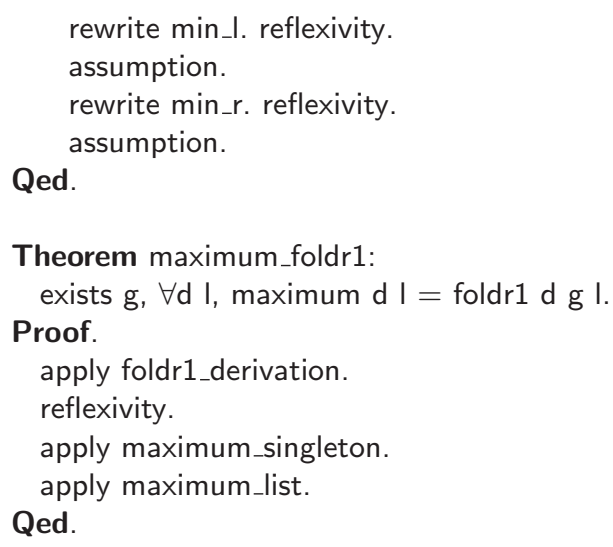

In this example, we import the content of several modules, in particular insert_sorting which defines a sorting function and foldr 1 which provides foldr1 and the associated theorem but for the list type of the Coq standard library (the content of this module is identical to the previous example, but a Require Import List command at the beginning of the file).

We first define maximum as the head of the list obtained by sorting the input list with the $\leq$ order on naturals (le in Coq and le_ge_dec for the proof that it is a decidable relation).

Then we prove the properties of the maximum when the list is a singleton and when it may be more than a singleton. Both lemmas are proved using mainly the tactics presented above, except unfold that replaces a term by its definition. We can then derive a better implementation of maximum using the theorem foldr1_derivation.

[13] is a quick yet longer introduction to Coq.

\section{Coq Tactics for Program Calculation}

This section starts with an overview of tactics we provide and with a demonstration of how they are used in calculation; it is followed by details about the implementation of the tactics in Coq.

\subsection{Overview of available tactics}

We provide a set of tactics to perform program calculation in Coq. We can use it in two ways: either we want to transform a program but we don't know what will the final result be; or we want to transform a program to another known program.

Let's take the example of maximum ${ }^{4}$ presented in introduction. We will first illustrate the case in which we don't know the final result with the singleton list case; then we will illustrate a calculation which just proves the equality between two known programs with the case in which the list has the form a::x

In the case of a singleton list, we want a function $f$ such that $\forall a$, maximum $d[a]=f a$; this is expressed by the type $\{f \mid \forall a d$, maximum $d[a]=f a\}$ of maximum_singleton in what is following.

Definition maximum_singleton : $\{f \mid \forall a d$, maximum $d[a]=f a\}$. Begin.

\footnotetext{
${ }^{4}$ maximum $\mathrm{d} \mathrm{I}$ is defined by hd $\mathrm{d}$ (sort $\left.\mathrm{I}\right)$ where $\mathrm{hd} \mathrm{d} \mathrm{I}$ is the function returning the head of the list $\mathrm{I}$, or $\mathrm{d}$ if $\mathrm{I}$ is an empty list.
} 


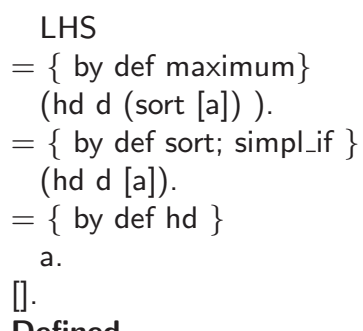

The Begin. tactic starts the session by doing some technical preparation of the coq system which will be detailed later (sect. 3.3).

Then the user specifies by the LHS tactic that he wants to transform the Left Hand Side of the equation maximum $d[a]=f$ a. If he had wanted to transform right hand side of the equation he would have used the RHS tactic, Or BOTH_SIDE tactic to transform the whole equation.

By using

$$
\begin{aligned}
= & \{\text { by def maximum }\} \\
& (\text { hd } d(\text { sort }[a])) .
\end{aligned}
$$

the user specifies that the left hand side should be replaced by hd d (sort [a]), and that this transformation is correct by the definition of maximum.

For the next transformation, the equality between the term hd d (sort [a]) and the term hd $d$ [a] cannot be proved by the only definition of sort: it also needs some properties over the relation "greater than" used in the definition of sort. The user-defined tactic simpl_if which, in a sense, helps to determinate the value of sort [a], is necessary. Actually, we can place here any necessary tactic sequence to prove the equality.

Once we've achieve a satisfying form for our program, we can use []. to end the transformation.

In case the list has the form $a:: x$, we want to prove that maximum $d(a:: x)$ is equal to if $a$ ? > (maximum $a x$ ) then a else maximum $a x$.

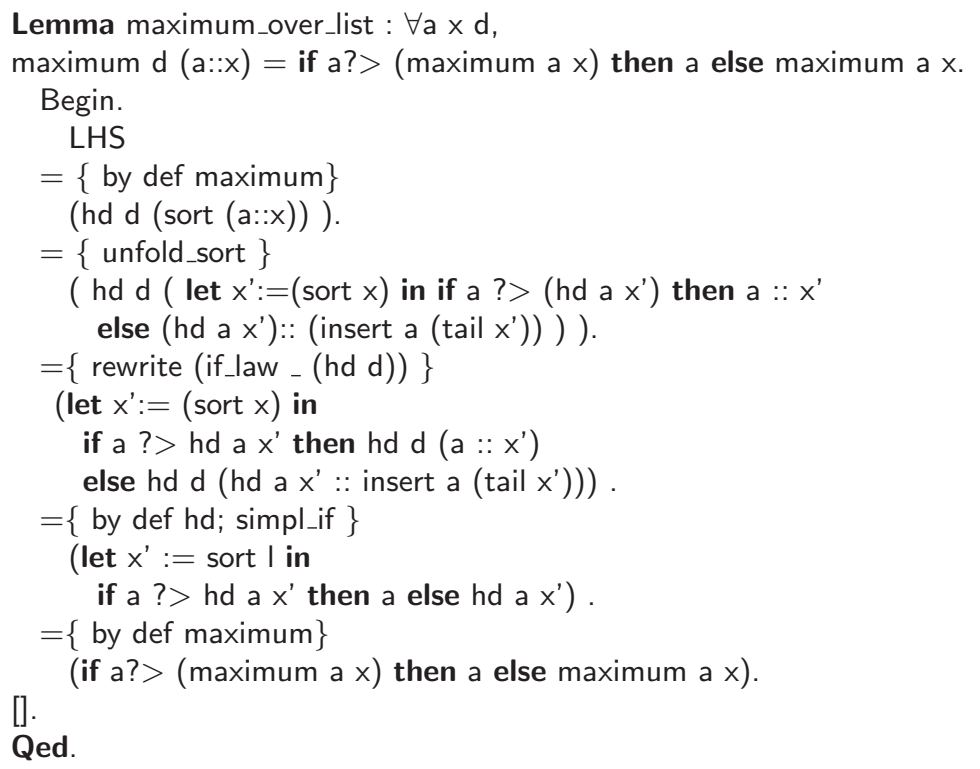


As previously we use Begin. to start the session. Then, left hand side of the equation is transformed using the definitions of programs and a program transformation law, if_law. This law states that for any function $\mathrm{f}, \mathrm{f}$ applied to an if $\mathrm{C}$ then $\mathrm{g} 1$ else g2 statement is equal to the statement if $\mathrm{C}$ then $\mathrm{f} g 1$ else $\mathrm{fg}$.

[] ends the proof if the two terms of the equation are convertible.

\subsection{More advanced use}

For the previous example we use the Coq equality but we can also use the system with a user-defined equality. For doing this we use the Setoid module from the standard library which allow to declare an equivalence relation. Let's take the extensional equivalence relation between function defined by

Definition ext_eq A B $(\mathrm{f}: \mathrm{A} \rightarrow \mathrm{B})(\mathrm{g}: \mathrm{A} \rightarrow \mathrm{B}):=\forall \mathrm{a}, \mathrm{f} \mathrm{a}=\mathrm{g}$ a.

Theorem ext_eq_refl, ext_eq_sym and ext_eq_trans which state that extensional_equality is respectively reflexive, symmetric and transitive can be proved easily. With setoid we can declare extensional equality as a user-defined equality by the following code :

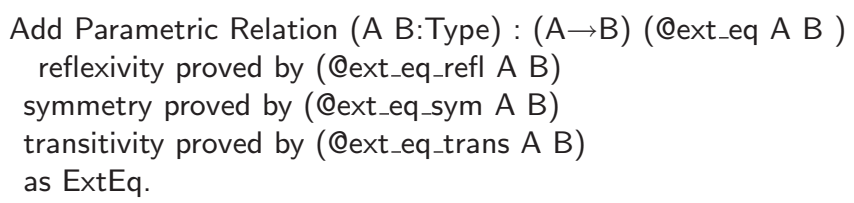

Afterward, we will denote @ext_eq A B f1 f2 by f1 == f2, the arguments A and B being declared implicit.

Once we have declared our relation, it can automatically be used by tactics like reflexivity, symmetry, transitivity or rewrite which are normally used with Coq equality.

However, if we know that $f 1==f 2$, then for any $F, F$ 1 can be rewritten into $F$ f2 by the tactic rewrite only if $\mathrm{F}$ is declared as a morphism (or is a syntactic composition of declared morphism) for $==$. For example the function composition

Definition comp $(A B C:$ Type $)(f: B \rightarrow C)(g: A \rightarrow B):=$ fun $(x: A) \Rightarrow f(g x)$,

is a morphism for $==$ for its two arguments $f$ and $g$. This means that

$\forall \mathrm{f} 1 \mathrm{f} 2 \mathrm{~g}, \mathrm{f} 1=\mathrm{f} 2 \rightarrow \mathrm{comp} \mathrm{f} 1 \mathrm{~g}==$ comp $\mathrm{f} 2 \mathrm{~g}$

and that

$\forall \mathrm{f} 1 \mathrm{f} 2 \mathrm{~g}, \mathrm{f} 1==\mathrm{f} 2 \rightarrow \operatorname{comp~} \mathrm{g} 1==$ comp g f 2.

This is implemented by:

Add Parametric Morphism A B C : (@comp A B C ) with

signature (@extensional_equality B C) $=\Rightarrow$ eq $=\Rightarrow$ (@extensional_equality $A$ C )

Proof. as compMorphism.

Qed.

Add Parametric Morphism A B C :( @comp A B C ) with signature $($ eq $)=\Rightarrow($ @extensional_equality $A B)=\Rightarrow($ Cextensional_equality $A$ C $)$

Proof. as compMorphism2.

Qed. 
And here is an example of use:

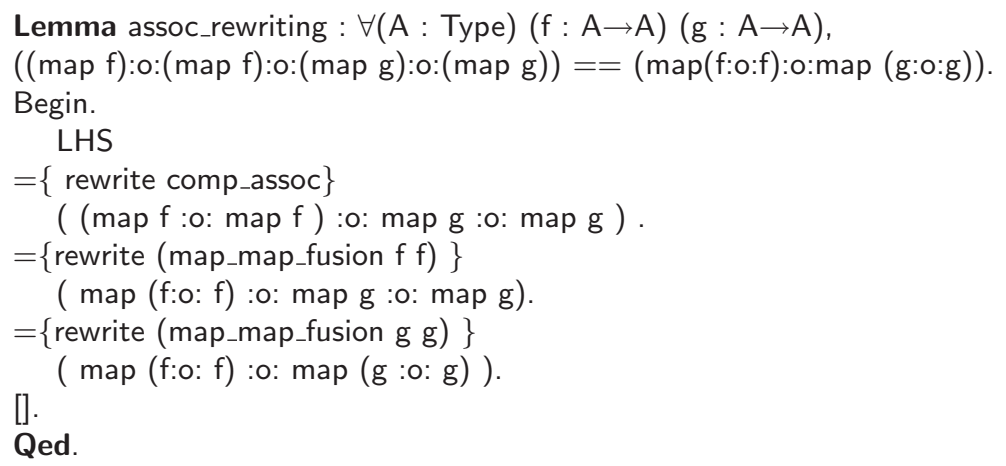

The :o: is a notation for the function composition, comp_assoc states that function composition is associative and map_map_fusion states that

map $f: 0:$ map $g=\operatorname{map}(f: 0: g)$.

We can see here that once the relation and the morphism are declared we can use the tactics as if it was the Leibniz equality.

\subsection{Behind the scene}

The Coq system allows the definition of syntax extensions for tactics, using Tactic Notation. These extensions associate an interleaving of new syntactic elements and formal parameters (tactics or terms) to a potentially complex sequence of tactics. For example, $=\{\mathrm{ta}\} \mathrm{t} 1$. is defined by

Tactic Notation (at level 2) "=" " $\{$ "tactic(t) " $\} " \operatorname{constr}(\mathrm{e}):=\ldots$.

tactic( $\mathrm{t})$ specify that the formal parameter $\mathrm{t}$ is a tactic and constr(e) specify that the formal parameter e has to be interpreted as a term.

The Begin. tactic introduces all premises and then inspects the goal. If the goal is existentially quantified, we use the econstructor tactic which allows to delay the instantiation of existantially quantified variables. This delaying permits to transform the goal until we know what value should have this variable.

The LHS and RHS tactics first verify that the form of the goal is R $a b$ and that $\mathrm{R} a \mathrm{~b}$ is a registered equivalence relation. Then they memorize a state used by the equivalence notation to know on which part of the goal it must work.

When registering the relation ext_eq as ExtEq, ExtEq is proved to be an instance of the type class Equivalence ex_eq. Type classes are a mechanism for having functions overloading in functional languages and are widely used in the module Setoid. They are defined in details in [14]; here, we only need to know that if there is a declared instance of the class Equivalence with parameter $\mathrm{R}$, Equivalence $\mathrm{R}$ can be proved only by using the tactic typeclasses eauto. We use this to check that the goal has the right form so that we can be sure that our transformations produce an equivalent program.

The notation $=\{\mathrm{ta}\} \mathrm{t} 1$., asserts that the term $\mathrm{t} 1$ is equivalent to the goal (or part of the goal, depending on the previously memorized state) and proves it using the tactic ta (followed by reflexivity). Then, the goal is rewritten according to the newly proved equivalence. 
The side of the equivalence which is transformed can be fully contained in the other side of the equation; thus, rewriting this term to an other could transform the other side of the equation that should be left untouched.

So we also have to protect the opposite side with the tactics LHS and RHS. To protect it, we use the tactic set which replaces a given term (here the side we want to protect) by a variable and adds a binding of this variable with the given term in the hypothesis.

Memorisation mechanism. Coq does not provide a mechanism to memorize information between tactic applications, so we introduce a dependent type which carries the informations we want to save. This type memo is defined by

Inductive memo (s: state) : Prop := mem : memo s .,

state being an inductive type defining the informations we want to memorize.

The memorization of a state s can now be done by posing mem _ : memo s. We define a shortcut

Tactic Notation "memorize" constr(s) := pose ( mem _ : memo s).

which abstracts the memorization mechanism. To access to the memorized information, we use pattern matching over hypothesis.

The main limitation of this mechanism is that the information is memorised until the current (sub-)goal is solved. We have no way to memorize information from one goal to another.

\section{Application: BMF in Coq}

In this section, we demonstrate the power and usefulness of our Coq tactics library for program calculation through a complete encoding of the lecture note on theory of lists (i.e., Bird-Meertens Formalisms for program calculation, or BMF for short) [4], so that all the definitions, theorems, and calculations in the lecture note can be checked by Coq and guaranteed to be correct. Our encoding ${ }^{5}$, about 4000 lines of Coq codes (using our library), contains about 70 definitions (functions and properties) and about 200 lemmas and theorems.

In our encoding, we need to pay much attention when doing calculation in Coq. First, we have to translate partial functions into total ones because Coq can only deal with total functions. Second, we should explore different views of lists, being capable of treating lists as snoc lists or join lists, while implementing them upon the standard cons lists. Third, we should be able to code restrictions on functions that are to be manipulated.

In the following, we give some simple examples to show the flavor of our encoding, before explaining how to deal with the above issues.

\subsection{Examples}

To give a flavor of how we encode BMF in Coq, let us see some examples. Using Coq, we do not need to introduce a new abstract syntax to define functions. Rather we introduce

\footnotetext{
${ }^{5}$ The code is available at our project page.
} 
new notations or define functions directly as those in Coq. For example, the following defines the filter function (and its notation) for keeping from a list the elements that satisfy a condition and the concat function for flattening a list of lists.

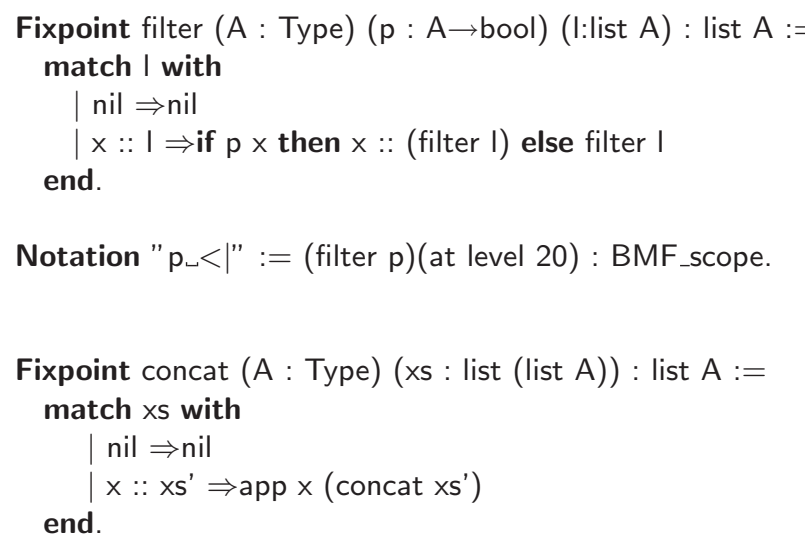

And the following shows how to prove the filter promotion rule [4] using our Coq tactics.

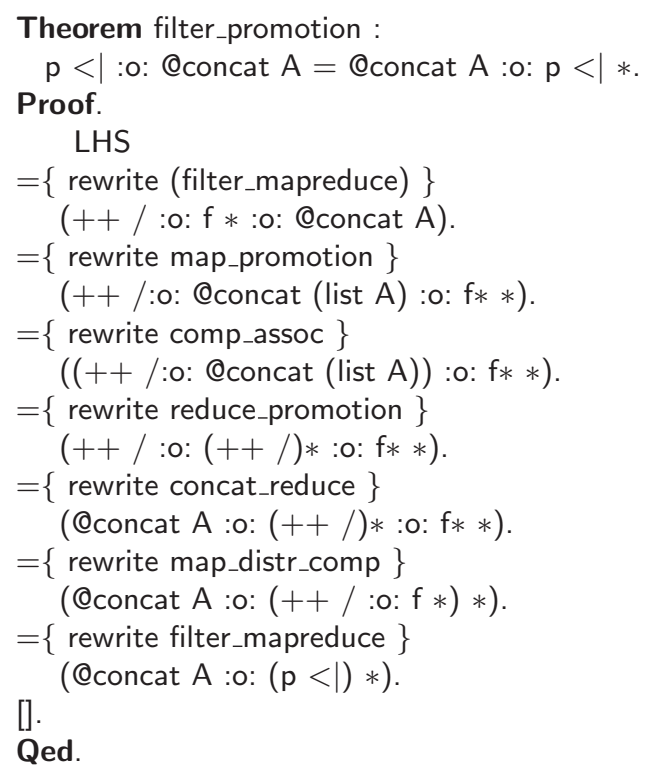

In the following, we discuss how we deal with partial functions, different views of lists, and properties imposed on functions.

\subsection{Implementation of partial functions}

In Coq, all functions must be total and should terminate in order to keep the consistency of the proofs; otherwise, arbitrary theorems will be proved and thus proofs will lose their meaning. However, there are many cases in which we want to use partial functions in programming. For example, the function that takes a list and returns the first element of the list is a partial function, because the function could not return any value if the list were empty. To implement this kind of functions as total functions, we may add "default value", so that if the input is out of the domain, then, the function will return the default value. For example, the "head" function is implemented as follows: 


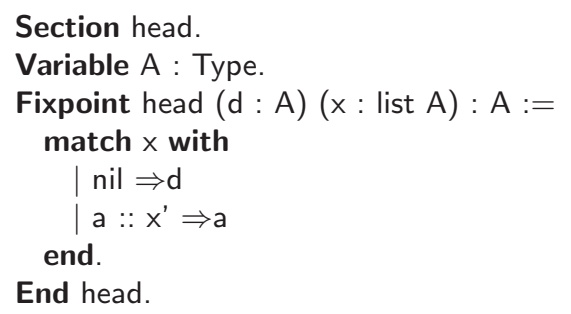

In fact, this definition is the same as the hd one in the standard Coq library. Alternatively, we may define this kind of partial functions with a condition. For example, we may define the "head" function as it is in the Section 2.

\subsection{Exploiting different views of a data type}

There are different views of lists in BMF. We usually define a list as an empty list or a "cons" of an element to a list as in Section 2. This captures the view of that a list is constructed by adding elements successively to the front of a list (we will call this view "cons list"). However, there are other views of lists. For example, the "snoc list" is a view that a list is constructed by adding an element one by one to the end of an empty list, and the "join list" is another view that a list is constructed by concatenation of two shorter lists.

Adopting different views would make it easy and natural to define functions or to prove theorems on lists, so we exploit these views based on the cons list by implementing functions (or properties) on snoc lists or join lists based on those on snoc as lists follows.

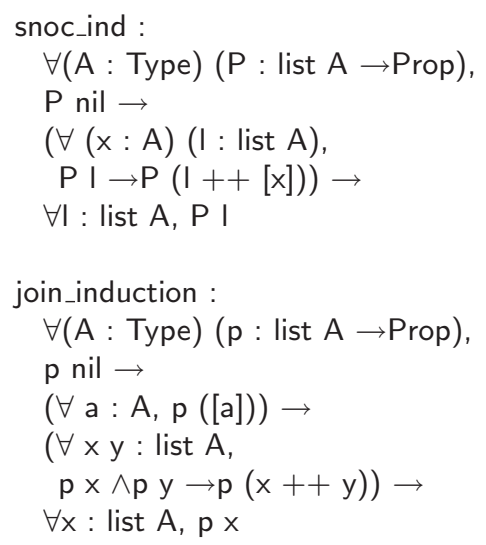

By implementing in this way, we can use useful induction principles on the snoc list and the join list.

To be concrete, let us explain the theorem that reflects the definition on the snoc list. Consider the following fold function defined on the cons list.

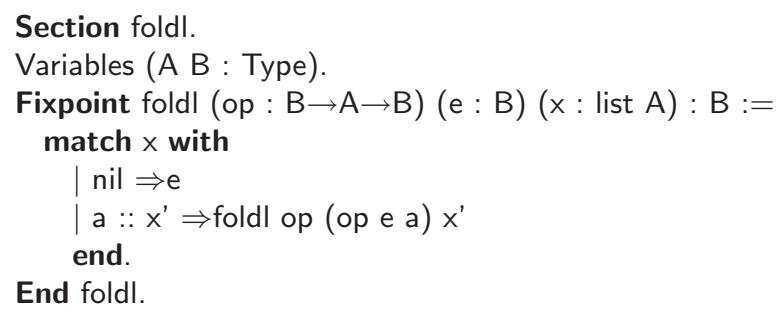


In fact, this fold can be more naturally defined over the snoc list.

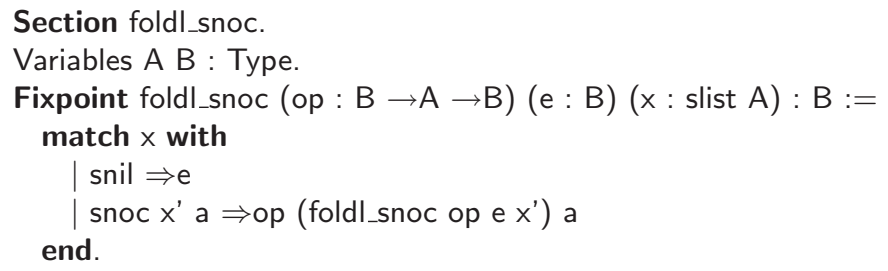

Actually, many proofs of theorems in BMF follow this fact. However, it is difficult to define such foldl_snoc function on cons lists. To resolve this problem, we introduce the following theorem instead:

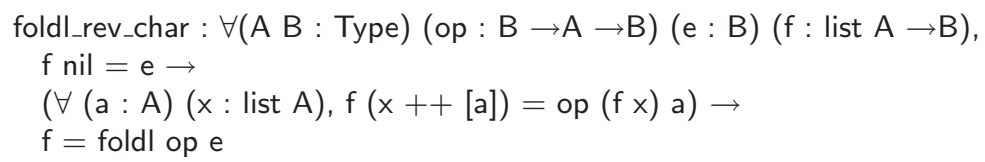

where we are allowed to use " $x++[a]$ " to denote "append $x$ (cons a nil)", which is not allowed in usual function definitions. This theorem means that it is sufficient to prove " $\mathrm{f}$ nil $=\mathrm{e}$ and $f(x++[a])=o p(f x)$ a for all $x$ and $a$ " in order to prove " $f=$ foldl op $e$ ". This theorem can be used to replace the above definition of foldl_snoc.

\subsection{Imposing constraints on a higher-order function}

In many cases, we have to define some computation patterns (higher-order function) parametrized with some functions (operators) that satisfy some property. For example, the reduce operator behaves like foldl op e, except that it requires that op and e form a monoid in the sense that op is associative and e is the identity unit of op.

To impose such monoidic constraints on the operators of reduce, we define it as follows.

Section reduce_mono.

Variables (A : Type)

$($ op : $A \rightarrow A \rightarrow A)(e: A)$.

Definition reduce_mono $(\mathrm{m}:$ monoid op $\mathrm{e})$ :

list $\mathrm{A} \rightarrow \mathrm{A}:=$

foldl op e.

End reduce_mono.

This reduce_mono exploits the fact that a proof is a term in Coq. Now if the associative operator doesn't have the identity unit in general, we can define the following reduce1 instead, by changing the constraint of reduce_mono.

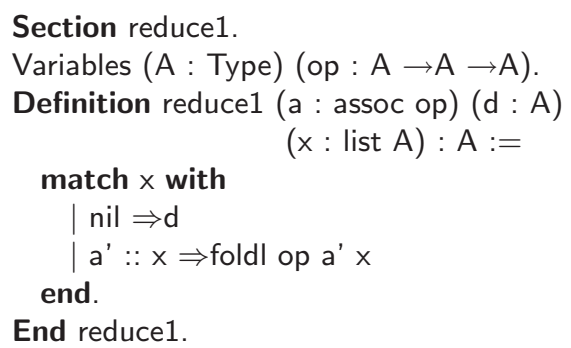


This reduce1 takes a term of type assoc op, where assoc op is a dependent type that denotes op is associative. Because it is natural to view reduce1 as a partial function defined on a non-empty list, we can implement it with a "default value" as mentioned in Section 4.2. In other words, if a list is nil, reduce1 returns the default value, otherwise reduce1 is defined in terms of foldl.

\section{Related Work}

There are many systems $[8-10,15]$ that have been developed for supporting program calculation. Unlike the existing systems, our system is implemented upon the popular and powerful theorem prover Coq with two important features. First, our implementation is light-weighted with only 200 lines of tactic code in Coq. Secondly, our system allows the rich set of theories in Coq to be used in program calculation for free.

Our work is much related to AoPA [16], a library to support encoding of relational derivations in the dependently typed programming language Agda. This follows the line of research for bridging the gap between dependent types and practical programming [17-19]. With AoPA, a program is coupled with an algebraic derivation whose correctness is guaranteed by the type system. However, Agda does not have a strong auto-proving mechanism yet, which would force users to write complicated terms to encode rewriting steps in calculation. Therefore, we turn to Coq. We have exploited Ltac, a language for programming new tactics for easy construction of proofs in Coq, and have seen many advantages of building calculational systems using Coq. First, the Coq tactics can be used effectively for automatic proving and automatic rewriting, so that tedious calculation can be hidden with tactics. Secondly, new tactics can coexist with the existing tactics, and a lot of useful theories of Coq are ready to use for our calculation. Third, the system on Coq can be used in a trial-and-error style thanks to Coq's interaction mechanism.

The basic idea of implementing equational reasoning in Coq, follows those that have been implemented in Agda [19]. Augustsson [17] has proposed a similar syntax for equality reasoning, with automatic inference of congruences.

A mecanism to describe equational reasonning is available in C-Zar (Radboud University Nijmegen) [20]. This language is a declarative proof language for the Coq proof assistant that can be used instead of the Ltac language to build proofs. It offers a notation to reason on equation but it is limited to Leibniz equality and doesn't allow to transform terms existantially binded.

\section{Conclusion and Future Work}

In this paper, we propose a Coq library to support interactive program calculation in Coq. As far as we are aware, this is the first calculation system built upon Coq. Our experience of using the library in encoding the Bird's theory of lists in Coq shows the usefulness and power of the library.

Our future work includes adding theorem about programs calculation and tactics to automate proof of correctness of program transformation and extension of the library to program refinement. Indeed, our system currently imposes restrictions on the relation between transformation by forcing it to be an equivalence. In the future, we could add the possibility to explicitely use relation that are not equivalence but refinement relation. 


\section{References}

1. Bird, R.: Constructive functional programming. In: STOP Summer School on Constructive Algorithmics, Abeland. (September 1989)

2. Kaldewaij, A.: Programming: the derivation of algorithms. Prentice-Hall, Inc., Upper Saddle River, NJ, USA (1990)

3. Bird, R., de Moor, O.: Algebras of Programming. Prentice Hall (1996)

4. Bird, R.: An introduction to the theory of lists. In Broy, M., ed.: Logic of Programming and Calculi of Discrete Design, Springer-Verlag (1987) 5-42

5. Gibbons, J.: Algebras for Tree Algorithms. D. Phil. thesis, Programming Research Group, Oxford University (1991) Available as Technical Monograph PRG-94. ISBN 0-902928-72-4.

6. de Moor, O.: Categories, relations and dynamic programming. Ph.D thesis, Programming research group, Oxford Univ. (1992) Technical Monograph PRG-98.

7. Hu, Z., Takeichi, M., Chin, W.N.: Parallelization in calculational forms. In: 25th ACM Symposium on Principles of Programming Languages (POPL'98), San Diego, California, USA, ACM Press (January 1998) 316-328

8. Smith, D.R.: KIDS - a knowledge-based software development system. In Lowry, M.R., Mccartney, R.D., eds.: Automating Software Design, Menlo Park, CA, AAAI Press / The MIT Press (1991) 483-514

9. de Moor, O., Sittampalam, G.: Generic program transformation. In: Third International Summer School on Advanced Functional Programming. Lecture Notes in Computer Science, Springer-Verlag (1998)

10. Yokoyama, T., Hu, Z., Takeichi, M.: Yicho: A system for programming program calculations. Technical Report METR 2002-07, Department of Mathematical Engineering, University of Tokyo (June 2002)

11. Bertot, Y., Casteran, P.: Interactive Theorem Proving and Program Development - Coq'Art: The Calculus of Inductive Constructions. Springer Verlag (2004)

12. : The Coq Proof Assistant. http://coq.inria.fr

13. Bertot, Y.: Coq in a hurry (2006) http://hal.inria.fr/inria-00001173.

14. Sozeau, M., Oury, N.: First-Class Type Classes. In: TPHOLs'08. (2008)

15. Visser, E.: A survey of strategies in rule-based program transformation systems. J. Symb. Comput. 40(1) (2005) 831-873

16. Mu, S.c., Ko, H.s., Jansson, P.: Algebra of programming in agda: Dependent types for relational program derivation. J. Funct. Program. 19(5) (2009) 545-579

17. Augustsson, L.: Cayenne - a language with dependent types. In: In International Conference on Functional Programming, ACM Press (1998) 239-250

18. McBride, C.: Epigram: Practical programming with dependent types. In Vene, V., Uustalu, T., eds.: Advanced Functional Programming. Volume 3622 of Lecture Notes in Computer Science., Springer (2004) $130-170$

19. Norell, U.: Dependently typed programming in agda. In Kennedy, A., Ahmed, A., eds.: TLDI, ACM (2009) $1-2$

20. Corbineau, P.: A declarative language for the coq proof assistant. In Miculan, M., Scagnetto, I., Honsell, F., eds.: TYPES '07, Cividale del Friuli, Revised Selected Papers. Volume 4941., Springer (2007) 69-84 